\section{THE PREVENTION AND TREATMENT OF POSTOPERATIVE INTESTINAL OBSTRUCTION.*}

DANIEL H. CRAIG, M.D.

Surgeon to Out-Patients, Free Hospital for Women. BOSTON.

In three previous papers ${ }^{1}$ I have reported the results in the study of this subject, and I am led to speak of it again at this time not alone because my continued study of the subject convinces me of its soundness and great value, which view is shared by many who have employed my suggestions, but more especially because $I$ desire to benefit by the discussion of this matter by the great field embraced only in the membership of this organization. To review the action of eserin salicylate on the intestine I shall quote from my first paper:

To prevent any occurrence it must first be thoroughly understood. It was, therefore, first necessary to understand how and why celiotomy produced paresis. This question is entirely elucidated by the peculiar anatomy and physiology of the intestine. With both the anatomy and physiology of the intestine every one here is familiar in a general way, and assuming this I shall only intrude on your time those facts not so generally known.

Anatomically we have to do with the longitudinal and circular coats of unstriped muscles with Auerbach's plexus of nerves between them and Meissner's plexus inside the circular coat. The unique effects produced on the intestine by its ex. posure to the irritation accruing from the lowered temperature and manipulations incident to celiotomy are largely owing to this peculiar nervous anatomy. The intestines, while unquestionably influenced both as to motor and vasomotor conditions by the central nervous system, through the splanchnics and vagi, are none the less essentially autonomous. It has many times been experimentally demonstrated that the ganglia comprised in Auerbach's and Meissner's plexuses are fully capable of complete reflex functions, so that perfectly normal motor equilibrium is easily and permanently maintained after com. plete severance of every slightest connection with the central nervous system. However, it is probable that in the human celiotomy patient we are dealing with a much more complex problem than in animals under experimentation, for it seems probable that the chilling and contact irritation cause an excitation of the splanchnics, which creates powerful reflex spinal inhilitory impulses while it at the same time produces a degree of over-stimulation of the intrinsic reflex ganglia which results in speedy ex.haustion.

Bayliss and Starling, ${ }^{2}$ who have very recently thoroughly investigated this subject, say: "The tendency of physiologists during the last thirty years has been to deny to peripheral ganglia or collections of ganglia any reflex function at all comparable to those possessed by the brain and spinal cord functions, that is to say, which include co-ordination as well as mere reflexion of impulses. The facts we have brought forward, however, show beyond doubt that the local nervous structures in the gut have this power of co-ordination, of directing one kind of influence along one path and another kind of influence along another path, the results being a 'purposive' response directed to the propulsion of the food down along the alimentary canal. Auerbach's plexus is, in fact, a local nervous system with two reflexes, inhibition and augmentation, and one function, the propulsion of food." The two reflexes, acting in cooperation, give rise to the phenomena which constitutes the basis of what is known as "The Law of the Intestine," namely, "If cerebrospinal reflexes be excluded, excitation at any point

* Read in the Section on Obstetrics and Diseases of Women of the American Medical Association, at the Fifty-eighth Annual Session, June, 1907.

1. 'The Prevention of Postoperative Intestinal Paresis and Adhesions, Am. Jour. of Obs., April, 1904. Postoperative Intestinal Paresis. A Further Contribution to Its Prevention. Am. Jour. of Obs., September, 1904. 'The After-Treatment of Abdominal Sections with Eserin Sallcylate. N. Y. Med. Jour., March 18, 1905.

2. Bayliss and Starling: The Movements and Innervation of the Small Intestine, Am. Jour. of Physiology, xxiv, 99. of the gut excites contraction above and inhibition below." In normal human beings under operation, however, the splanehnics and vagi are still operative, except in so far as they may be affected by air, temperature, trauma and anesthetic, and the problem is, therefore, less simple. The most marked influence exerted by the central nervous system through these nerves seems to be limited to the control of the vasomotor influences and the more distinct control of the ends of the alimentary canal, as, for example, swallowing and defecation. Courtard and Guyon ${ }^{3}$ say, however, that contraction and dilatation of the two muscular coats determined by stimulation of the splanchnics are entirely independent of any vasomotor influence, being the same whether the vagus is cut or its peripheral end stimulated. Moreover, according to Bayliss and Starling, ${ }^{2}$ the splanehnics are bearers of tonic inhibitory influences to the intestine. "These inhibitory impulses are reinforced by reflex inhibitory influences dependent on stimulation of the sensory nerves." This last sentence, taken in conjunction with the following quotation from the same authors, namely, "The most striking method of producing reflex inhibition is stimulation of the intestine itself. If one or both splanchnics be intact the slightest stimulus applied to the intestine, even a gentle handling of the gut, suffices to produce a reflex inhibition of the whole length of the intestine," shows quite clearly how intestinal paresis may result from the most simple exploratory celiotomy in the hands of the most expert. Mall, ${ }^{4}$ moreover shows that this inhibitory influence of the splanchnics is superfluous, as the contraction of the intestine continues independently of the central nervous system; the walls are filled with systems of nerves which, no doubt, perform functions independent of even the solar plexus. "We may admit that the whole intestine lives, to a slight extent, quite an independent life."

The foregoing shows quite abundant cause for paresis and yet it is possible that there are still further causes active during celiotomy, for Dastre and Morat say that asphyxia causes intestinal anemia (hence lessened intestinal activity) and restoration of oxygen produces the inverse effect. Therefore, it is quite possible that a prolonged or too profound anesthesia may play a certain rôle in intestinal paresis. There, too, Mall* has shown that peristalsis is more active after a meal and at rest during the night, therefore the preoperative cleansing of the intestinal canal favors paresis by removing the natural stimulus of intestinal contents. Various investigators have proved that when normal muscular action may be prevented in localized areas of the intestine, whether it be as spasm or as relaxation, or both in different areas, nothing so quickly re-establishes the normal rhythm of both the pendulum (Cannon's rhythmic segmentation) and propulsive mechanisms as the establishment of the normal peristaltic wave in the normal portion of the gut. Under this influence the spasm will relax and the dilated bowel contract.

One further point only. Postoperative intestinal paresis is probably due to a dual influence. Inlibition due to a stimulation of the splanchnic fibers and exhaustion due to over-stimulation and hence fatigue of the ganglia in Auerbach's and Meissner's plexuses. To regulate peristalsis we must then comteract the stimulated spinal reflexes acting through the splanchnics and restore the energy to the intestinal nerve end. ing and muscles. In a word, this constituted my problem.

As, detailed in my original paper, many months spent in painstaking trials showed that by means of eserin salicylate these results could be achicved. To show how it accomplishes this purpose permit me again to quote this time Professor Wood. ${ }^{*}$ He says $:^{7}$

It has no apparent local irritant action in therapeutic doses. Absorption and elimination are rapid, the latter principally by the kidneys. No sensible general effects are perceptible after full therapeutic doses of calabar bean, except it be slight weakness and dislike for muscular exertion. The physiologic

3. Courtard and Guvon: Archives de Physiologie, 1897.

4. Mall: Johns Hopkins Hosp. Reports, 1, 37, 1896.

5. Dastre and Morat: Archives de Physiologie, 1882 to 1884.

6. H. C. Wood: Therapeutics, Eleventh Eid., 223.

7. The italies are mine. 
action of calabar bean has suggested its use in spasmodic affections, in atony of the muscular coats of the bowel, and in various diseases of the eye. In constipation dependent on muscular relaxation and as an addition to laxative pills we have found it very useful. A Hiller strongly indorses the value of the extract in ehronic intestinal atony, after or during a catarrh, and in the convalescence from fever, etc., and in constipation with fatulence, in meteorism, ete. One-thirtieth of a grain of either alkaloid may be considered to be the full therapeutic dose. Intestinal peristalsis is primarily much increased by the action of calabar bean. After poisoning there is first a stage of exceedingly active movements in the bowels; then spasmodic, tetanic contractions of the intestine occur so that the caliber is very much diminished and finally relaxation and dilatation take place. After death the vermicular move. ments are found very much lessened or altogether abolished. The action of calabar bean on the intestine appears to be peripheral, due to the contact of the poison in the blood with the muscle fibers or the nerve elements in the walls of the bowel, for Westermann found that extirpation of the cardiac ganglia had no effect on the action of the drug, but that tying $t^{\prime} \mathrm{e}$ mesenteric and celiac arteries, before poisoning, prevented any increase in peristalsis. Probably there is an increase of the intestinal secretions

Owing to its sedative effects on the spinal reflexes Wood classifies it with spinal motor-depressants.

On reviewing these qualifications it is readily seen that eserin salicylate meets all demands. Its routine employment by myself, and latterly by many others, in all cases where not contraindicated or where not omitted for purposes of comparative observation, has demonstrated its absolute harmlessness, even in children. Owing to its depresso-motor action it is able to counteract the spinal inhibitory impulses carried to the intestine by the irritated splanchnics. Also acting directly to sustain and stimulate the intrinsic intestinal musculature or its nerve supply it combats or overcomes this source of paresis.

Continued employment of this drug with a wide study of its literature, especially the work being done by Oppenheim, von Noorden and others in its use as a treatment of intestinal paresis already established, together with personal experience in such employment of eserin on established cases of postoperative intestinal paresis seen in consultation, has led me to formulate original ideas on the entire subject of postoperative intestinal obstruction, which I hereby submit.

Be it understood, however, that under the caption of postoperative I include only such cases as are directly influenced by the recentness of the operation, thus being rendered quite an independent problem in etiology, diagnosis, prognosis and treatment. Cases of postoperative intestinal obstruction occurring so long after operation as to require no unusual treatment and to offer no unusual problems in diagnosis are postoperative only in an etiologic sense.

The etiology of postoperative intestinal obstruction in accordance with the foregoing limitations must be very recent and the inception of the complication must occur under the watchful care of surgenn and nurse. Preexisting and ordinary etiologic factors. such as bands, diverticulæ, hernial orifices, etc., can ordinarily be possitively eliminated owing to the surgeon's recent and exact knowledge of the conditions within the abdomen and pelvis. The factors to be considered are then reduced, with few exceptions, to paresis, volvulus, thrombosis of the intestinal vessels, adhesions in conjunction with kinks. and slight local infection.

Boise, ${ }^{8}$ under organic causes. discusses adhesions ex-

8. Eugene Boise: Post-Operative Intestinal Obstruction, Med. News, July 18,1896 . isting prior to operation and unrelieved by the operation. These cases deserve consideration as such only when it can be clearly shown that the operation was the immediate determining factor in causing those bands to produce obstruction at that particular time or when the obstruction shall supervene so closely on the operation that a secondary operalion for its relief carries an unusual degree of danger because of the recentness of the original operation. 'The other causes enumerated by Boise are: (1) A tonic intestinal spasm; (2) true intestinal paralysis; (3) formation of new adhesions. Regarding spasm he says: "After nearly all abdominal operations, even of moderate severity, there is transient obstruction, the evidence of which is intra-abdominal pain, more or less spasmodic in its nature and generally referred to a point at or near the seat of greatest violence." This symptom complex he attributes to exaggerated (or even spasmodic) peristaltic action. My own jdeas will be brought out later.

True intestinal paresis and its method of origin have been so fully treated by myself in earlier papers and by many authoritative physiologists that it requires no special elucidation here.

Volvulus, aside from its peculiar postoperative relationship, is, of course, generally confined to the sigmoid flexure and is fully understood. After the manipulation of the intestinal coils incident to a celioiomy a volvulus is possible in the small intestine as well and especia!iy in connection with recent alhesions, as will be shown later.

A subject not so extensively known is that of postoperative intestinal obstruction due to thrombosis of the intestinal vessels. Marsh ${ }^{9}$ has shown that direct injury may give rise to occlusion of the mesenteric artery, and Nothnagel $^{10}$ (page 280) quotes him to agree with his statements. Oppolzer, Faber and Kaufmann have reported cases in which thrombotic occlusion of the trunk of the superior mesenteric artery involved the entire small intestine (with the exception of the middle and upper horizontal portion of the duodenum) and in addition the cecum and ascending and transverse colon. When lesser vessels become occluded a continuous segment of the intestine suffers most commonly in the lower jejunum or ileum, although, of course, any part of the intestine may be involved, corresponding necessarily with the distribution of the occluded vessel. While the mesenteric arteries are not anatomically terminal arteries in action during life, the experiments of Litten and Faber show the superior mesenteric to act like one in regard to hemorrhagic infarction after occlusion.

After such experimental occlusion of the superior mesenteric artery $\mathrm{Welch}^{11}$ and $\mathrm{Mall}^{12}$ found first tonic contraction and anemia for two to three hours; then relaxation and venous hyperemia followed by hemorrhagic infarction in from three to six hours. Litten and Faber believe that the inferior mesenteric artery is terminal neither anatomically nor functionally.

Perhaps this is the proper place to consider the fact which Nothnagel ${ }^{10}$ (p. 292) brings out, namely, that the circulation of dyspneic blood through the intestine may produce fairly active peristaltic movements. On the other hand, under certain circumstances, dyspneic blood has been known to have the reverse effect-inhibiting intestinal movements. But more important than the direct effect of dyspneic blood on the bowel wall is

9. Marsh : Brit. Med. Jour., 1902, i, 694

10. Nothnagel: Nis. of the Intestines and I'eritoneum.

11. Welch: Allbut's Srstem of Med., vol. il, 180 .

1:. Mall. Johns Hopkins Hosp. Leports, vol. i, 1359 
the result of poor blood supply, e. g., in heart disease or portal obstruction, on the nerve supply, especially the intrinsic ganglia. Etiologically this suggests that postoperative intestinal paresis may be more commonly expected in cases in which Auerbach's and Meissner's plexuses have long been poorly nourished, as they would be more than ordinarily affected by the operation and the dyspneic blood of anesthesia.

Leichtenstern ${ }^{13}$ has shown that decided flexions and abnormal positions of the intestines, such as commonly must obtain after celiotomies, especially where gauze packings and partitionings have been employed, need not necessarily interfere with the passage of their contents, provided only there are no adhesions causing kinks in the canal; therefore, such flexions and abnormal positions can only cause postoperative intestinal obstruction when allowed to persist until the adhesions have formed.

Nothnage $^{10}$ (p. 475) describes the causation of postoperative intestinal obstruction by recent (new) adhesion glueing together the adjacent sides of a looped intestine throughout their entire contiguity and thus producing a sharp angulation. On the other hand, where two portions of a large loop become attached to each other at a single point no obstruction may be directly resultant but volvulus becomes extremely easy, especially in the sigmoid.

Nothnagel, in closing his discussion on this subject, says :

The form of peritonitis leading to these intestinal adhesions is usually produced by some surgical operation, for instance, herniotomy or ovariotomy. In other cases the peritonitis is not traumatic but merely remains localized.

Albrecht, ${ }^{14}$ Rokitansky, ${ }^{15}$ Kundrat, ${ }^{16}$ Schnitzler $^{17}$ and very recently Finney ${ }^{18}$ have called attention to compression of the duodeno-jejunal junction by the mesenteric border or more particularly by the superior mesenteric artery when the small intestine is drawn or gravitates into or toward the pelvis sufficiently far to put traction on this artery. This may be considered an etiologic factor after operation for Cesarean section, ovariotomy or the removal of other large tumors which had caused overstretching of the abdominal walls, and especially in the use of the Fowler posture in septic cases, but in all instances seems easily avoidable as a postoperative complication. Albrecht ${ }^{14}$ attributes this descent in some cases to the downward pressure of a distended stomach, but should be considered inexcusable when so readily prevented by gastric lavage.

Another etiologic factor mentioned by Nothnagel (pp. 544, 545) is omental cords, the only requisite being the adherence of a given point on the omentum to some relatively fixed point. This constitutes a powerful argument against stitching the omentum to cover raw surfaces and shows the necessity for the prevention of unnecessary adhesions in general.

The diagnosis of postoperative intestinal olstruction within the first few days resolves itself practically into the differentiation between mechanical obstruction, paresis, septic peritonitis and shock.

13. Leichtenstern: Verengerungen, Verschliessungen und Lageveranderungen des Darms. v. Ziemssen's Handb. d. Spec. Path. u. Therap., 1878, ii, 2nd half.

14. Albrecht: Ueber arterio-mesenterialen Darmverschluss an $d$. Duodeno-Jejunalgrenze, Virchow's Archiv. clvi.

15. Rokitansky: Ueber Darmschniirungen, Oesterreichische Med. 15. Rok

brb., $1836, x$

16. Kundrat: Ueber eine Seltene Form d. inneren Incarcerationen, Wien. med. Wochschr., 1891.

17. Schnitzler: Tur. Symptomatologie d. Darmsarterienverschluss, Wien. med. Wochschr. 1901, Nos. 11, 12.

18. Hinney: Boston Med. and surg. Jour., 1906-7.
In the very early stages differentiation is probably always impossible, and even if the presence of leucocytosis always indicates peritonitis, any two or three or all of these four conditions may coexist. Therefore, while our intimate and recent knowledge of intra-abdominal conditions enables us to eliminate an acute exacerbation of all forms of chronic occlusion, all neoplasms, diverticulæ, etc., the ordinary postoperative phenomena render the diagnosis still more than ordinarily difficult.

Potain $^{19}$ has called attention to the fact that if the abdomen collapses during expiration it is because the diaphragm is immovably fixed and the respiration entirely thoracic, and so indicates peritonitis rather than intestinal obstruction unless, of course, in the later stages, when they coexist. But in our observation of respiratory phenomena we must always include frequent and thorough examinations of the thoracic viscera, as the diagnosis of peritonitis and even of obstruction has been made in cases of pleurisy and pneumonia.

The employment of air distension of the lower bowel for diagnosis of obstruction is either contraindicated or rendered valueless by the concomitant conditions within the first few days after a celiotomy and should very rarely, if ever, be employed.

'The differentiation of intestinal obstruction and peritonitis are difficult only in the early stages, but after a celiotomy this is the very time when a quick and correct decision is necessary to save life. The etiologic factors of both conditions-for, of course, the paresis is the most common form observed-have recently been active. Even the most careful surgeon can never be certain that his asepsis, never absolute, has been sufficient. Then since this differentiation is so difficult, why not elimis nate, if possible, the element of paresis?

Vomiting comes on earlier in peritonitis than in intestinal obstruction, but vomiting loses its distinctive value as a symptom of either in a recently anesthetized patient.

Visible peristalsis can scarcely be expected to aid us, inasmuch as it is usually a symptom of an acute obstruction following a long-standing chronic stenosis and, moreover, the abdominal dressings, wound, etc., render it difficult of observation even if present.

The character and location of pain are of little diagnostic value as ordinarily observed immediately after celiotomy, as practically every such abdomen in which neither peritonitis nor obstruction assumes importance as complications is the seat of much pain.

Persistently localized pain in the region of the sigmoid should, of course, warn us of a possible volvulus at that point. But as such volvulus is very early accompanied by peritonitis, the characteristic localization is very early lost.

In thrombosis of the mesenteric vessels it is entirely possible for the patient to have passed the contraction stage and to have reached the period of relaxation, which could never be differentiated from intestinal paresis before recovering from the anesthetic. That this statement is not overestimating the possibilities of rapid advance of this condition $I$ would quote Nothnagel (p. 287), who says that death may occur in from twentyfour to forty-eight hours. Mayland ${ }^{20}$ has reported a personally observed case of postoperative thrombosis of the mesenteric vessels.

Regarding Boise's contention that a majority of the symptoms of postoperative intestinal obstruction are

19. Potain: Circumscribed Subdiaphragmatic Peritonitis, Int.

Clinics, iit, 41, October, 1895.

20. Mayland: Brit. Med. Jour., 1901, vol. ii, p. 1454. 
due to intestinal spasm, I must say that I can not interpret the data so as to agree with his deductions nor does my personal observation tend to this view. Quite the contrary.

If the intestinal spasm were extensive a retracted abdominal wall should be found. Yet such has never been reported. Dr. Murphy's case of lead poisoning seems to me out of the reckoning.

Localized spasm involving only a relatively small portion of the bowel could be diagnosed only by the colicky pain, and since the publication of Dr. Boise's paper I have carefully observed cases in which no special preventive treatment was instituted and have failed to find any such pain, and, moreover, in listening to the intestinal sounds as practiced by Cannon ${ }^{21}$ in his study of the intestinal movements, absolute silence has been invariable.

Dr. Boise adduces much to support his contention from the writings of authoritative physiologists, while in my earlier papers I have extensively quoted the same authors to prove the exact opposite. In what I shall call untreated cases, bearing reference only to suggestions which I wish to submit later, I have found the pain steady, hard, greatest at and about the site of greatest trauma, increased by movement, respiration and pressure, but not spontaneously exacerbated in a way to suggest enterospasm. ${ }^{22}$

The subject of differentiation might be elaborated so as to constitute alone a paper many times the proper length of such a paper as this. but I feel that even without the foregoing hints everyone realizes that this differentiation within the first few days after a celiotomy is always extremely difficult and often impossible, and agrees that any harmless measure which shall serve to render such differentiation unnecessary or at least easier should be employed.

The question of postoperative adhesions has been frequently discussed, and after the work of such men as Martin, Morris and others no apology nor defense is needed for the suggestion of a method for their prevention or destruction.

In my earlier papers my suggestion was that in cases not demanding, from the nature of the work done, complete intestinal rest a hypodermic injection of atropin sulphate should precede the administration of the anesthetic, and that on the operating table, as soon as it could be determined that it was not contraindicated, as above, often only two to five minutes after the opening of the peritoneal cavity, a hypodermic injection of eserin salicylate in fresh solution be administered.

This lessens spinal reflex inhibition of peristalsis in which it is aided by the previously administered atropin. It sustains, by its action directly on the intestinal musculature, or the nerve terminations therein, the autonomous action of Auerbach's and Meissner's plexuses and prevents the transitory postoperative intestinal paresis commonly observed in patients not so treated. ${ }^{23}$

Constant employment of this method for six years leaves me more enthusiastic in its advocacy than at the

21. Cannon: Auscultation of the Rhythmic Sounds Produced by the Stomach and Intestines, Am. Jour. of Physiol., Oct. 2, 1905, xiv, 339 .

22 . It seems worthy of note that in so exhaustive a work, replete with editorial and translator's notes, as Stengel's translation of Rolleston's edition of Notbnagel's "Diseases of the Intestines and Peritoneum," in a very full discussion of the subjects of "Colic" and "Enterospasnim" no mention is made of laparotomy or exposure of the intestine as an etiologic factor of enterospasm.

?3. I would respectfully refer those who do not care to accep this apparently arbitrary statement to the arguments fully adduced in my earller papers. beginning, and the statements which follow are based on this observation.

Two arguments have been adduced against this employment of eserin. My friend, Dr. Stone," takes the ground that fatal intestimal paresis is too uncommon to necessitate its prophylaxis in every case. In a measure this is true, but when such a case occurs the surgeon's absolute impotence is too well known to need emphasis. Moreover, the prevention of the minor degree of postoperative paresis adds more to her comfort than many so much discussed doses of morphia. The other argument is that it prevents adhesions and thus diffuses septic material. Certainly no intelligent surgeon would employ it in a case in which a septic focus had been unavoidably left behind any more than he would in an intestinal resection. Such adhesions are conservative and invaluable, but nevertheless, as Reichel ${ }^{25}$ claims, so I believe that in every celiotomy a peritoneal infection too slight to demonstrate occurs and from this infection proceed peritonitis, intestinal paresis and adhesions. But these adhesions are pathology pure and simple, neither lefensive nor in any way salutary, and should be prevented, not to mention adhesions resulting from purely mechanical peritoneal irritation, raw surfaces, etc.

To recur once and finally to the question of enterospasm. Granting that my deductions may be incorrect and that enterospasm actually exists, physiologists show why and my cases show that the prophylactic use of cserin certainly relieves it. Various physiologists have proved that nothing so rapidly overcomes either a localized spasm or a localized area of paresis as an advancing peristaltic wave, and such waves eserin excites. This is borne out clinically by the fact that in cases : which eserin is used symptoms which Boise attributes to spasm do not appear.

The pain commonly observed after abdominal section I attribute first to trauma and secondly to the stretching of the peritoneal bowel investment by the gaseous distension of the bowel at a time when this peritoneal coat is abnormally sensitive.

The pain, perhaps more properly termed sensitiveness to pressure and bodily movements, due to trauma is comparatively slight and transitory and, needless to remark. is not influenced by eserin.

This brings us to the consideration of meteorism. Our first concern should be to learn why the paretic intestine becomes thus distended with gas. Nothnagel (p. 72) says: "Under normal physiologic conditions all the different movements of the intestine hitherto enumerated are caused solely by the irritating effect of its contents, whether chyme, feces or gas." Many physiologists have proved the correctness of this statement.

However, in the preparation of patients for celiotomy the strictly limited diet, together with cathartics, removes the material for a chyme bolus, and thus patients are denied this stimulus to peristalsis. In the administration of salines we see the exemplification of the use of the liquid lolus, but, as. I have pointed out, this has decided disadvantages. But in those cases in which the intestine has been practically emptied and in which salines have not been administered, peristalsis is urgently required and Nature makes an effort to supply the only remaining bolus possible, namely, gas. This, of course, is rendered the more easy owing to the stagnation resultant on the abeyance of peristalsis. Therefore, in the

24. Stone, I. S.: Abuse of Purgation Before and After Opera tion. Trans. Southern Surg. and Gyn. Asso., xvil, 64

25. Reichel: Zur Pathologie des Ileus u. Pseudolleus. Sitzungsber, d. Wiirzburger Med. Gesell, 1892, No, 7. 
light of the foregoing and fortified by circumstances later to be mentioned, I have come to look on this gaseous distension as conservative up to a certain point. It is only when the plexuses of Aucrbach and Meissner have become exhausted or the reflex inhibition of the splanchnics is greater than ordinary that such gaseous bolus fails to restore peristalsis. But it is in the borderline cases that our interference is most needed, because when the gas distension passes a certain point of tension it causes paralysis of the intestinal muscles just as the bladder or other hollow viscus may be paralyzed by overdistension.

Our prophylactic administration of eserin clinically prevents meteorism, as anyone may prove by intelligent observation. 'This I explain in two ways: Inasmuch as intestinal paresis is either entirely prevented or peristalkis is very early re-established, the physiologic necessity of bolus stimulation is entirely lacking, hence no meteorism is occasioned. This is, of course, facilitated by an absence of stagnation. Another explanation of the absolute absence of meteorism after the prophylactic use of eserin is the fact that, as stated by Woods, the eserin materially increases the secretion of the intestinal juices which serve to prevent putrefaction.

This absence of meteorism is, of course, greatly to be desired on account of the patient's comfort, but in addition it serves another salutary purpose, namely, the prevention of peritonitis. Kocher ${ }^{26}$ has shown, and in common with other well-known authorities is quoted, with agreement by Nothnagel, that the peritonitis which arises in connection with intestinal paresis must be studied from a special point of view, inasmuch as the walls of the intestine, especially those portions which lave become paralyzed and in which meteorism develops, become permeable to the bacteria present in the bowel lumen. As soon as these bacteria penetrate the peritoneal cavity peritonitis naturally develops. Kocher further declares that this permeability is not dependent on any one form of occlusion, but is a result of the stretching of the structures of the intestinal wall. If the correctness of this view be granted, how easily many cases of postoperative peritonitis in cases in which meteorism has been allowed to develop and in which the surgeon, his instruments, sutures or assistants have been considered unclean, are explained. And how important the prevention of such meteorism becomes.

Now leaving the question of paresis, let us consider those cases in which, owing to replacement of intestinal coils, the placing of gauze for walling off the operative field, or merely the manipulation of the intestine incidental to exploration, coils of intestine are left in abnormal relationships, twisted or kinked. Nothing can so readily straighten out those kinks and twists as an advancing peristaltic wave. 'This may be feebly simulated by forcing liquid through a kinked rubber tube. There, too, these kinks and twists, etc., are perpetuated by two factors, first, meteorism which so fills the abdomen as to prevent free adjustment of the intestinal interrelationship, and second, the formation of adhesions. Active peristalsis prevents both these factors and would seem therefore highly desirable.

But, in addition, Oppenheim, Fraser, von Noorden and others have shown that eserin is capable of curing many cases of well-established postoperative intestinal paresis and it is coming to be very commonly so used by those not yet employing it in the prophylactic manner.

26. Kocher : Ileus. Grunzgeb. d. Med. v. Chir., iv. Also, Deutsch Ztschr. f. Chir., viil.
Here, of course, the finest diagnostic discrimination must be used, as in mechanical obstructions other than as above noted escrin would act eren more harmfully than most of our ordinary cathartics.

To illustrate its use in the cure of postoperative intestinal paresis, I wish to briefly report one case of my own and two cases the data of which were furnished me by Dr. I. S. Stone, of Washington:

CAsE 1.-I was called on the telephone at 10:30 p. m. by Dr. Hayes of Brookline, who gave me the following

History.-Patient was operated on three days before and all efforts to move the bowel had proved futile. Respiration was much embarrassed by intense meteorism. She had been seen in consultation by three of the best surgeons in Boston, who concurred in the diagnosis of postoperative intestinal paresis. He desired me to see the patient at once. I advised him, accepting the diagnosis of the men who had seen her, and as he'r condition was desperate, not to wait for me but to give her 1/150 grain of atropin and 1/40 grain of eserin salicylate in fresh solution immediately and $I$ agreed to hold myself in readiness to see her if this first administration failed. Just one hour later Dr. Hayes called me again to say that in twentynine minutes after the administration of the eserin immense quantities of gas began to be expelled by the anus and that the patient was very comfortable. She made a good recovery.

The next two cases I will copy verbatim from Dr. Stone's report to me:

Case 2.-Patient F. U. G., aged 37 years, v-para, widow; induced abortion, resulting in right salpingo-oöphoritis.

operation.-Removal of right appendages. Peritonitis with extreme distension. Eserin salicylate gr. 1/20 given by hypodermic twenty-nine hours after operation. One hour later medium, dark, fluid stool. One-half hour later large, dark, fluid stool. Pulse and respiration improved. Sixteen hours later eserin salicylate gr. 1/20 with nitroglycerin gr. 1/100. Fifty minutes later large, dark, fluid stool. Ten minutes liter large, dark, fluid stool. No apparent depression.

CAsE 3.-Dr. I., age 43. The patient came for operation after his third attack of appendicitis from which he was just recovering. The present attack was associated with gastrointestinal catarrh. $\mathrm{He}$ is accustomed to the rather liberal use of alcohol and had a coated tongue and foul breath. $\mathrm{He}$ is corpulent, although very active and of a rather nervous temperament. After two days rest and "preparation," which included a thorough cleansing of the alimentary canal, operation on Dec. 5, 1904. The appendix was long and adherent, but had not ruptured. No accident nor complication of any kind during the operation or anesthesia. His pulse and temperature remained about normal during the convalescence. Vomiting set in during the afternoon after operation and persisted in spite of lavage and starvation until the end of the fourth day. Eserin salicylate gr. 1/30 hypodermically at 3 o'clock p. m. The patient did not know the nature of the drug but knew that it was for the relief of the vomiting. He declared that the reversed peristalsis was changed to normal in about thirty minutes and that the relief was prompt and very marked, in fact, complete. He was anxious to have a second dose at 10 o'clock and persuaded the interne on duty to give another $1 / 30$ grain at that hour. There were no further bad symptoms of any kind and he left the hospital in less than three weeks.

Finally, just a word about dosage. It must be remembered that eserin can overdo its good work and it is quite easy to cause meteorism and abdominal distension after celiotomy by completely exhausting the intestinal musculature by too large or too frequent doses. I use $1 / 40$ grain on the operating table and very rarely have occasion to repeat it. In the discussion of this subject one man said that in his experience he had found fully as much or even more abdominal distension after the use of eserin than without it. On investigation I found he was using gr. 1/20 to gr. $1 / 12$ for his initial prophylactic injection and repeated it at relatively frequent in- 
tervals. I repeated his techmic in three cases and invariably got intestinal exhausion with intense meteorism.

The keynote of success in the after-treatment of cases in which the prophylactic dose of eserin has been administered is letting the pat ent alone. Her abdomen is soft and comfortable, vomiting is minimized. Thirst is slight and cathartics and enemata are entirely unnecessary. As soon as her diet supplies sufficient intestinal residue her bowels will behave as any non-operative patient who is quiet in bed. In other words, the aftertreatment of such cases is purely expectant or symptomatic.

\section{SUCCESSFUL TREATMENT OF ACUTE POST- OPERATIVE ILEUS,}

BY INCISION AND DRAINAGE OF THE INTESTINE, WITH REPORT OF FOUR CASES. *

\section{FRANCIS D. DONOGHUE, M.D.} Boston.

Some paralysis of peristaltic activity usually follows surgical interference in the abdominal cavity. It is more common after operations on acute inflammatory or suppurative conditions, but may follow apparently slight operations with clean conditions of the peritoneum.

The milder forms are readily overcome by some of the usual postoperative procedures. Those cases which do not yield to ordinary treatment, however, and especially those cases accompanying localized or general peritonitis, present a most dangerous and trying complication.

Whatever may be the actual causative factors in the production of acute postoperative ileus, the practical results are of a mechanical nature, plus poisoning from the products of intestinal decomposition. This peristaltic inertia is often aggravated by adhesions between loops of intestines causing "kinking," or from slight adhesions between the omentum and some viscus or inflamed surface. These adhesions, not in themselves of a character and extent to cause trouble, may become of serious import in the presence of some intraintestinal factor.

\section{SYMPTOMS}

The symptoms of serious postoperative ileus develop early and in twenty-four to thirty-six hours may be well marked. In fact, some operations, while successful in removing a primary cause of trouble, do not cause amelioration of symptoms, so that the developing ileus forms a continuous picture with the condition which was the cause of interference.

Persistent vomiting, changing from a bilious to a stercoraceous character, should put one on guard. With this comes an increase in the abdominal distension which is not relieved by the use of enemata; neither is the vomiting controlled by gastric lavage.

As a rule, the temperature is not increased, unless the basis of the trouble is a spreading peritonitis. In the worst cases of ileus the temperature may be normal or even subnormal. The pulse, however, is a fairly reliable guide. Some hours (twenty-four to thirty-six) following recovery from the immediate operative shock and accompanied by increasing signs of non-restoration of

- Read in the Section on Obstetrics and Diseases of Women of the American Medical Association, at the Fifty-eighth Annusl Session. June, 1907. intestinal function, the pulse begins to increase in rapidity and to lose in character and quality.

\section{CLASSICAL SIGNS.}

The classical signs of obstruction, notably pain and dulness in the flanks, are obscured and the picture of shock, with persistent vomiting and increasing distension, is presented. As the distension and vomiting increase, profound shock appears which, in a patient unoperated on, would forbid operation.

In postoperative cases, however, a sign which might be accepted as a certain contraindication to operative interference, under ordinary conditions, points conclusively to further surgical intervention if the patient is to be saved.

The purpose of this paper is to point out a treatment that has been found serviceable in these severe cases. The other phases of the question are to be covered by more extended papers in this and other sections.

A certain number of cases of postoperative ileus, in spite of all varieties of treatment directed to non-secondary operation, do badly.

The treatment by the use of eserin ${ }^{1}$ and the treatment by physiologic doses of atropin may occasionally be found useful, but when cases have reached a critical state, mechanical treatment offers the best chance for recovery.

The value of intestinal drainage was recognized in the early days of abdominal surgery in connection with cases of diffuse peritonitis of appendicular origin.

Dr. Mixter ${ }^{2}$, of Boston, as far back as 1894, advocated, in certain cases of generalized peritonitis, the drainage of distended loops of intestine, either by puncture, or by puncture followed by the tying of a tube into the cecum. Dr. Greenough, of Boston, in 1904, in "Fnterostomy in Peritonitis," reviewed the subject from the time that Dr. Mixter published his first article in 1895 .

While the value of enterostomy in acute intestinal obstruction and in cases of slow developing chronic obstruction, which finally give rise to acute symptoms, has been well recognized, its value as a postoperative procedure is yet to be fully appreciated. Its value is threefold: 1. It affords a means for the escape of gas and liquicl feces. 2. It affords an opportunity, by the injection of solutions of magnesium sulphate, to provoke peristalsis at considerable distance from the point where the tube has been placed. 3. Stimulating fluids and nourishment can be introduced.

When nourishment or stimulating fluids are introduced through a tube placed at the "point of election" in the cecum, the fluids are rapidly absorbed, maintaining heart and kidney functions, while the lavage of the intestine from copious amounts of warm fluid, with or without the addition of a cathartic, tends to provoke peristalsis in the small intestine many feet away.

In all cases as a preliminary to drainage by the tube at the time of secondary interference, all loops of intestine which are markedly distended should be incised and the liquid contents and gas should be emptied out, followed by immediate closure with a continuous intestinal suture. Large amounts of gas and decomposing intestinal contents are thus eliminated and the intestine given an opportunity to resume its function, which was in part prevented by overdistension.

Introduction of salts into the lumen of the small in-

1. Ztschr. P. Gynak., March 5, 1904.

2. Boston Med. and Surg. Jour., Feb. 28, 1895.

3. Boston Med. and Surg. Jour., May 19, 1904. 